\title{
Testing of Selected Accuracy Parameters for the Single Axis Positioner at the Automated Workplace
}

\author{
Marek Vagaš ${ }^{1}$, Ján Semjon ${ }^{2}$, Alena Galajdová1, Dušan Šimšík ${ }^{1}$, Róbert Rákay ${ }^{1}$, Patrik Šarga ${ }^{1}$, Martin \\ Višňovský ${ }^{1}$ \\ ${ }^{1}$ Technical University of Košice, Faculty of Mechanical Engineering, Department of Automation and Human Machine \\ Interactions, Letná 9, 04002 Košice Slovakia, marek.vagas@tuke.sk \\ ${ }^{2}$ Technical University of Košice, Faculty of Mechanical Engineering, Department of Robotics, Letná 9, 04002 Košice \\ Slovakia.
}

\begin{abstract}
Although some authors realized various measurement techniques and relevant standards in the experimental verification, the existing contributions still did not mention more complex automated workplaces with industrial robotic arm participation. To solve this, we provide a different view of the interconnection between individual devices (positioner, robot, etc.) controlled by third-party methods (Siemens products). Also, to obtain the necessary effectiveness, we tested and verified selected accuracy parameters for the participating device component (positioner). The proposed work tries to fulfill expectations for a precise welding technology (to ensure simultaneous movements of both the industrial robotic arm and positioner) to achieve higher quality and productivity. However, the components are from different manufacturers.
\end{abstract}

Keywords: Positioner accuracy, automated workplace, welding operation, ISO 230-2, Siemens PLC.

\section{INTRODUCTION}

Nowadays, there are increasing demands for the automated devices' quality, accuracy, and speed (welding processes including). Although the robotic system itself shall be an accurate, flexible, and effective mechanical system, not every auxiliary device (positioner, for example) allows high adjustability in terms of variability of the motion required by the workpieces in production and non-production areas [1]. In terms of accuracy and repeatability of positioning, the relevant standard ISO 230-2:2006 contains proper reference scope to validate the correctness of the measurement chain experimental setup at the complex automated workplaces. It helps set the demands on device parameters, characteristics, and reliability of operation [2]. Instead of a new and expensive solution, finding another way in a mechanical device, such as a positioner. However, this approach is not entirely trivial because every manufacturing process still requires precise movements. If it is indispensable to add external axes to the automated workplace, a positioner (with at least one additional axis) is a correct replacement.

Moreover, producers push to implement their own (often closed and expensive) applications that prefer the simplicity of control and faster realization [3]. Instead of such an often costly solution, our proposal wants to offer a cheaper alternative by different manufacturers' devices used at one common space (workplace) with reliable, constant, accurate positioning [4]. Even though the previous works have made several significant contributions to developing such automated workplaces focusing on the experimental verification of their equipment accuracy evaluation, some crucial aspects were still not mentioned.

The previous works focused on the configuration with a tilting-rotary table or a universal head. Most of them were designed based on multiple setups with the utilization of laser instruments [5]. Length gauge sensors from HEIDENHAIN play a role in quality assurance or any application where fast, reliable, and accurate measurement is required. Nevertheless, the measuring accuracy through standard length gauge on different configurations has not been fully considered yet. Among previous contributions, only Schwenke et al. [6] and Kwang et al. [7] have proposed using a length gauge to identify positioner accuracy to aim for geometric error measurement.

However, these configurations cannot be set as a general principle for implementation to the SMEs because the measurement method is dependent on geometric errors of five-axis machines. The reason is that we can choose this solution's configuration only to measure the calibrated threedimensional (3-D) probe and a precision sphere. Therefore, the implementation into the SMEs welding operations with the auxiliary device (in the form of a positioner) is worthy of a more detailed study. 


\section{SUBJECT \& METHODS}

The accuracy and repeatability of positioning in the proposed automated workplace must meet criteria defined in the ISO standard 230-2:2006 that specify test procedures [8]. The tests serve to measure the relative displacements between the subject (component) that holds the tool (industrial robot) and the subject (component) that contains the workpiece (positioner). The position to which the moving part is programmed to move is called "target position $P_{i}$." We can describe it as:

$$
P_{i}(i=1 \text { to } m)
$$

where:

$i$ - identifies the particular position among other selected target positions along or around the axis,

$m$ - number of positions in 1 cycle.

The measured position reached by the moving part on the $j$ th approach to the $i$-th target position is called "actual position $P_{i j .}$. We describe it as:

$$
P_{i j}(1 \text { to } m ; j=1 \text { to } n)
$$

where:

$j-$ serial number of the approach to the target,

$n$ - total number of unidirectional approaches in both the positive (increasing) and the negative (decreasing) directions in the same position.

The actual position reached by the moving part minus the target position is a deviation of position (positional deviation) $x_{i j}$. Calculated as:

$$
x_{i j}=P_{i j}-P_{i}
$$

The term "unidirectional" refers to the series of measurements in which the approach to a target position is realized in the same direction along or around the axis. It must calibrate the kinematic system. The control system of the robotic arm at the automated workplace must follow and take external kinematics into account during the path planning. The symbol $\uparrow$ signifies a parameter derived from a measurement made after approaching the positive direction and $\downarrow$ the negative direction, e.g., $x_{i j} \uparrow$ or $x_{i j} \downarrow$. The arithmetic mean of the positional deviations that we obtain by a series of $n$ unidirectional approaches to position $P_{i}$ is a term defined as the mean unidirectional positional deviation at a position:

$$
\overline{x_{\imath}} \uparrow=\frac{1}{n} \sum_{j=1}^{n} x_{i j} \uparrow
$$

and

$$
\bar{x}_{\imath} \downarrow=\frac{1}{n} \sum_{j=1}^{n} x_{i j} \downarrow
$$

The arithmetic mean of the mean unidirectional positional deviations $x_{i} \uparrow$ and $x_{i} \downarrow$ obtained from the two directions of approach at position $P_{i}$ is defined as "mean bi-directional positional deviation at a position, $\bar{x}_{i}$," and we calculate it as:

$$
\overline{x_{\imath}}=\frac{\overline{x_{l}} \uparrow+\overline{x_{\imath}} \downarrow}{2}
$$

The value of the difference between the mean unidirectional positional deviations obtained from the two directions of approach at position $P_{i}$ is "reversal value at a position, $B_{i}$."

$$
B_{i}=\overline{x_{\imath}} \uparrow-\overline{x_{\imath}} \downarrow
$$

Maximum of the absolute reversal values $\left|B_{i}\right|$ at all target positions along or around the axis is represented as "reversal value of an axis, $B$."

$$
B=\max \left[\left|B_{i}\right|\right]
$$

The arithmetic mean of the reversal values $B_{i}$ at all target positions along or around the axis is called "mean reversal value of an axis, $\bar{B}$," and calculated as:

$$
\bar{B}=\frac{1}{m} \sum_{i=1}^{m} B_{i}
$$

The estimator of the standard uncertainty of the positional deviations we obtain through a series of $n$ unidirectional approaches at position $P_{i}$ is called "estimator for the unidirectional axis repeatability of positioning at a position, $s_{i} \uparrow$ or $s_{i} \downarrow$.” We can calculate it as:

$$
s_{i} \uparrow=\sqrt{\frac{1}{n-1} \sum_{j=1}^{n}\left(x_{i j} \uparrow-\bar{x}_{\iota} \uparrow\right)^{2}}
$$

and

$$
s_{i} \downarrow=\sqrt{\frac{1}{n-1} \sum_{j=1}^{n}\left(x_{i j} \downarrow-\overline{x_{l}} \downarrow\right)^{2}}
$$

Bi-directional repeatability of the positioning at position $R_{i}$ can be calculated as:

$$
R_{i}=\max \left[2 s_{i} \uparrow+2 s_{i} \downarrow+\left|B_{i}\right| ; R_{i} \uparrow ; R_{i} \downarrow\right]
$$

Range derived from the estimator for the unidirectional axis repeatability of positioning at position $P_{i}$ using a coverage factor of 2 is called "unidirectional repeatability of positioning at a position $R \uparrow$ or $R \downarrow$."

$$
R \uparrow=\max \left[R_{i} \uparrow\right]
$$

and

$$
R \downarrow=\max \left[R_{i} \downarrow\right]
$$

The maximum value of the repeatability of positioning at any position $P_{i}$ along or around the axis is described as " $b i$ directional repeatability of the positioning of an axis $R$ " and calculated as: 


$$
R=\max \left[R_{i}\right]
$$

The difference between the algebraic maximum and minimum of the mean unidirectional positional deviations for the one approach direction $x_{i} \uparrow$ or $x_{i} \downarrow$ at any position $P_{i}$ along or around the axis is "unidirectional systematic positional deviation of the axis $E \uparrow$ or $E \downarrow$, calculated as:

$$
E \uparrow=\max \left[\bar{x}_{\imath} \uparrow\right]-\min \left[\bar{x}_{\imath} \uparrow\right]
$$

and

$$
E \downarrow=\max \left[\overline{x_{\imath}} \downarrow\right]-\min \left[\overline{x_{\imath}} \downarrow\right]
$$

Difference between the algebraic maximum and minimum of the mean unidirectional positional deviations for both approach directions $x_{i} \uparrow$ and $x_{i} \downarrow$ at any position $P_{i}$ along or around the axis are called "bi-directional systematic positional deviation of an axis $E$ " and calculated as:

$$
E=\max \left[\overline{x_{\imath}} \uparrow ; \overline{x_{\imath}} \downarrow\right]-\min \left[\overline{x_{\imath}} \uparrow ; \overline{x_{\imath}} \downarrow\right]
$$

The difference between the algebraic maximum and minimum of the mean bi-directional positional deviations $x_{i}$ at any position $P_{i}$ along or around the axis is "mean bidirectional positional deviation of an axis $M$," and calculated as:

$$
M=\max \left[\bar{x}_{l}\right]-\min \left[\bar{x}_{l}\right]
$$

Unidirectional accuracy of positioning of an axis is determined below:

$$
A \uparrow=\max \left(\overline{x_{\imath}} \uparrow+2 s_{i} \uparrow\right)-\min \left(\overline{x_{\imath}} \uparrow-2 s_{i} \uparrow\right)
$$

and

$$
A \downarrow=\max \left(\overline{x_{\iota}} \downarrow+2 s_{i} \downarrow\right)-\min \left(\overline{x_{\iota}} \downarrow-2 s_{i} \downarrow\right)
$$

The following equation determines the bi-directional positional accuracy of an axis:

$$
\begin{gathered}
A=\max \left(\overline{x_{\imath}} \uparrow+2 s_{i} \uparrow ; \bar{x}_{\imath} \downarrow+2 s_{i} \downarrow\right)- \\
\quad \min \left(\bar{x}_{\iota} \uparrow-2 s_{i} \uparrow ; \bar{x}_{\imath} \downarrow-2 s_{i} \downarrow\right)
\end{gathered}
$$

\section{THE EXPERIMENTAL SETTING}

The industrial robotic arm contains the standard control system AX-C based on the Win NT system and I/O card with $32 \mathrm{I} / \mathrm{O}$ inside (Fig.1.). The positioner is controlled through the Drive module SINAMICS S 120, and the robot control system is set as a master to the positioner [9].

PLC (Siemens SIMATIC S7-300 completed with the I/O modules) sends the required speed and rotation values according to the signal coming from the industrial robotic arm and sends them to the drive module of the positioner PROFIBUS interface [10].

The complete testing arrangement of the automated workplace consisted of the hardware equipment starting with the robotic arm, positioner and Siemens control components, HEIDENHAIN devices, and related software (SINAMICS MICROMASTER STARTER).
The standard length gauge MT 25 was used with the highly accurate setting at the reference point of the workpiece in all three axes $(X, Y$, and $Z$ ), vertically and horizontally. Clean measuring points are a prerequisite for high process reliability [11]. Digital readout serves as a numeric display that reads the signals generated at the automated workplace from the length gauge.

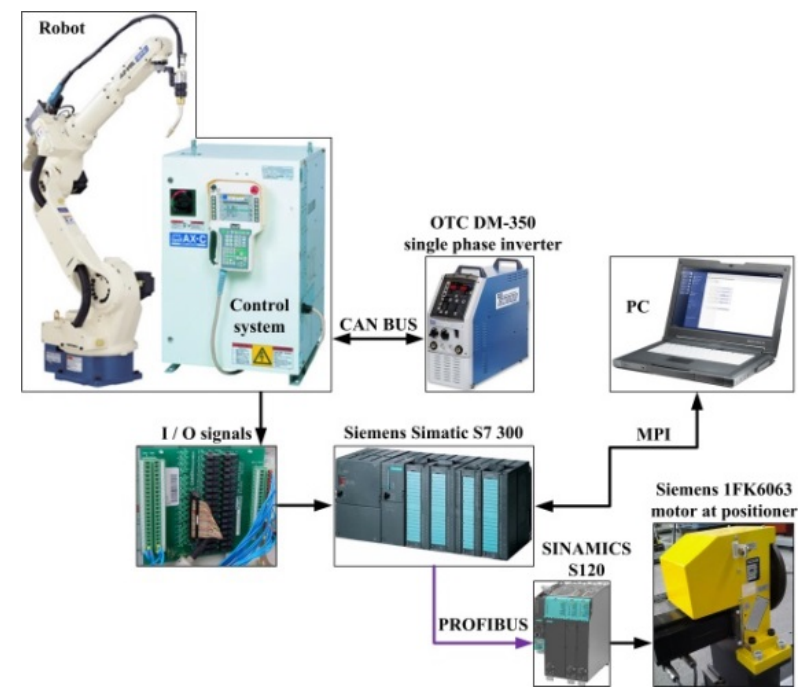

Fig.1. Scheme of interconnection between the components.

\subsection{VERIFICATION OF THE POSITIONER ACCURACY}

The positioning accuracy and the repeatability measurement of the rotary axis must be evaluated respecting the mentioned ISO 230-2. The verification for assessing positioning accuracy and repeatability for the rotary axis ranges up to 360 degrees.

Positioner accuracy connected with the rotary axis error measurement contains two categories. The first one is the methodology based on rotary axis positioning error measurement.

In contrast, the second includes methods of spatial orientation of a rotary axis with the relation to linear axes, determination of the actual rotary axis, and evaluation of the stability axis positioner [12].

We count the technique employing a HEIDENHAIN length gauge enabling measurement of rotary axes as the most popular method for measuring rotary axis positioning errors.

The length gauge attachment is stable and records motions following the changes of rotary axis angular position of the positioner in respect to definitions of the standard.

The motion sets maintain the measuring angle between the length gauge and the rotary table (Fig.2.). The error effectively differs between the target position, determined by the length gauge, and the actual angular position of the tested axis of a positioner. This method enables verification of the axis positioning accuracy, which is essential for the welding process.

The transformation block (from the rotary movement to the translational movement) consists of an aluminum profile (40x40) that is firmly connected (by two screws M12) to the rotary module of the positioner. 


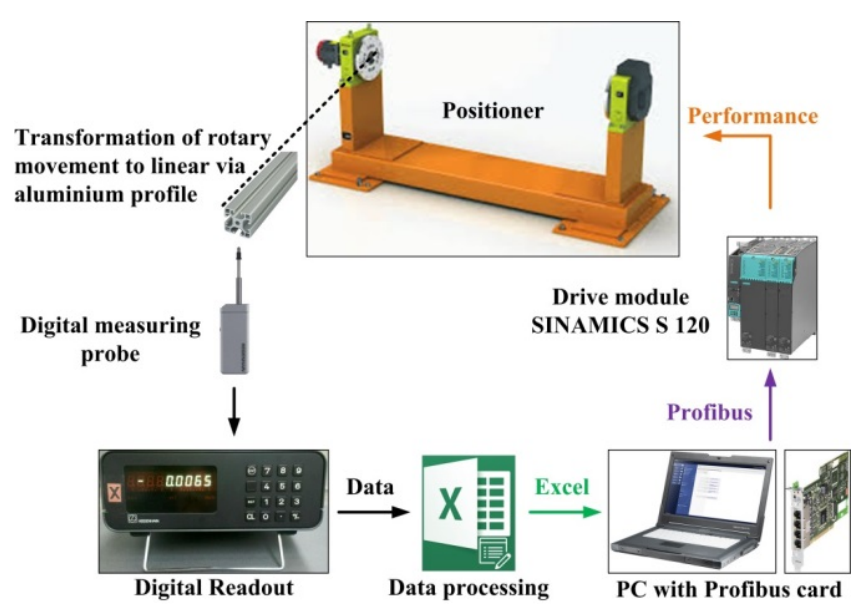

Fig.2. Measurement chain (scheme) for the positioner accuracy.

Correctness of the transformation block realization proves experimental tests for verifying the aluminum profile deviation to avoid production inaccuracies. We implemented and tested such solutions to measure the industrial robotic arm characteristics [13], [14], and [15] with the reliability of such technical property.

\subsection{CONDITIONS FOR MEASUREMENT}

On the positioner for measuring the linear axis of travel up to $2 m$, we selected a minimum of five target positions per meter and an overall minimum of five target positions. Measurements contain all target positions according to the standard test cycle [16].

We approached each target position seven times in each direction. The critical parameter has determined two-way positioning accuracy, i.e., the difference between the programmed position and the set of points achieved. We applied the following rules:

- Movement is realized from the reference point to the first position towards the reference, and then moving back to the reference point,

- Measurement is utilized in nine reference points and thirteen positions in total towards the reference,

- All desired measurement points must use this method in the plus and minus direction,
- The physical measurement construction (with load) is realized by the aluminum profiled bar with the weight situated at a $500 \mathrm{~mm}$ distance from the axis of rotation (Fig.3.).

- We chose a weight with the value of $31.5 \mathrm{~kg}$ to simulate the heaviest part allowed for the tested welding workplace $(30.2 \mathrm{~kg})$.

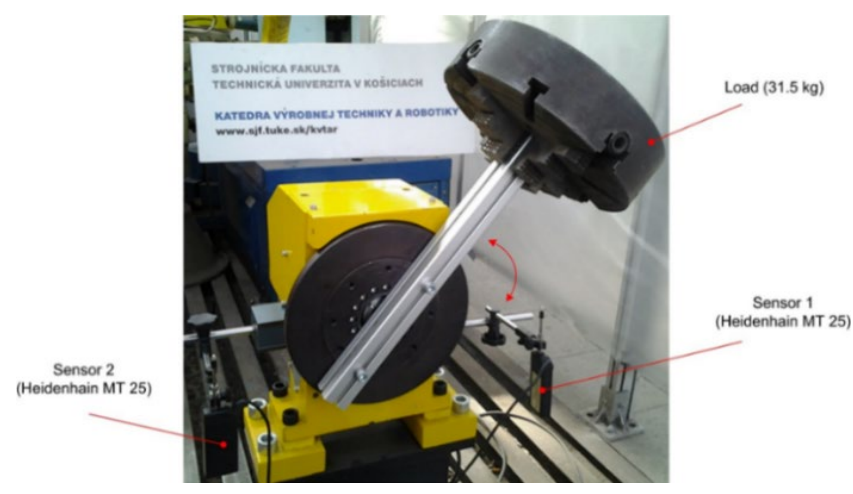

Fig.3. Measurement with load based on the aluminum profile attachment.

\section{Results}

We performed the analysis of test results in Microsoft Excel. The obtained measured data from testing are in Table 1., Table 2., and Table 3. The measuring process was performed with the positioner in the range $0^{\circ}$ to $360^{\circ}$. The position $0^{\circ}$ represents 12 hours (as it is in the case of an analog clock). The data value obtained was measured clockwise (plus) and counterclockwise (minus) every $45^{\circ}$.

The most critical position is $135^{\circ}$ (in both directions, target position 4) and $-360^{\circ}$ (position after one rotation of the positioner, counterclockwise, position 9). We obtained the highest value of measured data at mentioned $-360^{\circ}$ position ($9 \mu \mathrm{m})$. We measured the next highest value of the data at the $-135^{\circ}$ position ( $-9.5 \mu \mathrm{m}$, counterclockwise direction).

These data values indicate positioner overload, mainly in a position between $135^{\circ}$ and $365^{\circ}$. The values obtained from these data confirm repeated measurements and recalibration of the HEIDENHAIN length gauge.

Table 1. Positional deviations $x_{i j}(\mu \mathrm{m})$.

\begin{tabular}{|c|c|c|c|c|c|c|c|}
\hline \multirow{2}{*}{$\begin{array}{l}\text { Number of target positions } i \\
\text { Target position }\end{array}$} & & \multicolumn{2}{|c|}{1} & \multicolumn{2}{|c|}{2} & \multicolumn{2}{|c|}{3} \\
\hline & & \multicolumn{2}{|c|}{$\mathbf{0}^{\circ}$} & \multicolumn{2}{|c|}{$45^{\circ}$} & \multicolumn{2}{|c|}{$90^{\circ}$} \\
\hline Approach direction & & $+\uparrow$ & $-\downarrow$ & $+\uparrow$ & $-\downarrow$ & $+\uparrow$ & $-\downarrow$ \\
\hline Approach number & & & & & & & \\
\hline & 1 & 2 & 2 & 1 & -1.5 & -2 & 0.5 \\
\hline & 2 & 3.5 & -4 & 0.5 & -2 & -4 & -5.5 \\
\hline & 3 & 3 & -3 & 0.5 & -5 & -4.5 & -3.5 \\
\hline & 4 & 1 & -3 & 0.5 & -3 & -3.5 & -3 \\
\hline & 5 & 0.5 & -2.5 & 1 & -6.5 & -2.5 & -3.5 \\
\hline & 6 & 4 & 2.5 & 0.5 & -7.5 & -3 & -3 \\
\hline & 7 & 6 & 2 & 0.5 & -5 & -2.5 & -4 \\
\hline
\end{tabular}


Table 2. Positional deviations $x_{i j}(\mu \mathrm{m})$.

\begin{tabular}{|c|c|c|c|c|c|c|c|}
\hline \multirow{3}{*}{$\begin{array}{l}\text { Number of target positions } i \\
\text { Target position } \\
\text { Approach direction }\end{array}$} & & \multirow{2}{*}{\multicolumn{2}{|c|}{$\frac{4}{135^{\circ}}$}} & \multirow{2}{*}{\multicolumn{2}{|c|}{$\frac{5}{180^{\circ}}$}} & \multicolumn{2}{|c|}{6} \\
\hline & & & & & & \multicolumn{2}{|c|}{$225^{\circ}$} \\
\hline & & $+\uparrow$ & $-\downarrow$ & $+\uparrow$ & $-\downarrow$ & $+\uparrow$ & $-\downarrow$ \\
\hline Approach number & & & & & & & \\
\hline & 1 & -1 & -1.5 & 0.5 & 1.5 & -2.5 & -3 \\
\hline & 2 & -2.5 & -4 & 1.5 & 2.5 & -3.5 & -3.5 \\
\hline & 3 & -4.5 & -6.5 & 0.5 & 6.5 & -4.5 & -4 \\
\hline & 4 & -6.5 & -2 & 3 & 5 & -3.5 & -6.5 \\
\hline & 5 & -8 & -5 & 1.5 & -2 & -4.5 & -1.1 \\
\hline & 6 & -5.5 & -9.5 & 5 & -4 & -5 & -1.1 \\
\hline & 7 & -5.5 & -9 & 5 & -4.5 & -5.5 & -1.3 \\
\hline
\end{tabular}

Table 3. Positional deviations $x_{i j}(\mu \mathrm{m})$.

\begin{tabular}{|c|c|c|c|c|c|c|c|}
\hline \multirow{3}{*}{$\begin{array}{l}\text { Number of target positions } i \\
\text { Target position } \\
\text { Approach direction }\end{array}$} & & \multicolumn{2}{|c|}{7} & \multicolumn{2}{|c|}{8} & \multicolumn{2}{|c|}{9} \\
\hline & & \multicolumn{2}{|c|}{$270^{\circ}$} & \multicolumn{2}{|c|}{$315^{\circ}$} & \multicolumn{2}{|c|}{$360^{\circ}$} \\
\hline & & $+\uparrow$ & $-\downarrow$ & $+\uparrow$ & $-\downarrow$ & $+\uparrow$ & $-\downarrow$ \\
\hline \multirow[t]{8}{*}{ Approach number $\quad j$} & & & & & & & \\
\hline & 1 & 0.5 & 0 & -1.5 & 0.5 & -1.5 & -2 \\
\hline & 2 & -2.5 & -2.5 & -3.5 & 3 & -1 & -3 \\
\hline & 3 & -4.5 & 4.5 & -3.5 & 0 & -1.4 & -6.5 \\
\hline & 4 & -5 & 1.5 & -2 & -0.5 & -1.3 & -6.5 \\
\hline & 5 & -5.5 & 0 & -2 & 2.5 & -1.2 & -9 \\
\hline & 6 & -6 & -2.5 & -2 & 4.5 & -1.3 & -9 \\
\hline & 7 & -6.5 & -5.5 & -0.5 & 2.5 & -1.3 & -1.5 \\
\hline
\end{tabular}

Important derived parameters calculated as defined in standard ISO are in Table 4.

Table 4. Positioning accuracy and the repeatability of the rotary positioner axis $(\mu \mathrm{m})$.

\begin{tabular}{|c|c|c|c|c|c|c|c|c|c|}
\hline Serial number of target position $i$ & 1 & 2 & 3 & 4 & 5 & 6 & 7 & 8 & 9 \\
\hline $\begin{array}{l}\text { Mean bi-directional positional } \\
\text { deviation at a position } \bar{x}_{\mathrm{i}}\end{array}$ & 1 & -1.9 & -3.1 & -5.1 & 1.6 & -3.5 & -2.4 & -0.2 & -3.3 \\
\hline Reversal value at a position $B_{i}$ & 3.7 & 5 & 0 & 0.6 & 1.7 & -1.2 & -3.6 & -3.9 & 4.1 \\
\hline $\begin{array}{l}\text { Estimator for the unidirectional } \\
\text { axis repeatability of positioning at } \\
\text { a position } \mathrm{s}_{\mathrm{i}} \uparrow \text { (uncertainty) }\end{array}$ & 1.7 & 0.2 & 0.8 & 2.2 & 1.8 & 1 & 2.3 & 1 & 0.1 \\
\hline $\begin{array}{l}\text { Estimator for the unidirectional } \\
\text { axis repeatability of positioning at } \\
\text { a position } \mathrm{s}_{\mathrm{i}} \downarrow \text { (uncertainty) }\end{array}$ & 2.7 & 2.1 & 1.7 & 2.9 & 4.0 & 1.8 & 3.0 & 1.7 & 2.9 \\
\hline $\begin{array}{l}\text { Unidirectional repeatability } \\
\text { of positioning at a position } \mathrm{R}_{\mathrm{i}} \uparrow\end{array}$ & 7.0 & 0.9 & 3.3 & 8.8 & 7.2 & 3.8 & 9.1 & 4.0 & 0.6 \\
\hline $\begin{array}{l}\text { Unidirectional repeatability } \\
\text { of positioning at a position } R_{i} \downarrow\end{array}$ & 10.6 & 8.4 & 6.7 & 11.7 & 16.0 & 7.3 & 11.9 & 6.7 & 11.8 \\
\hline $\begin{array}{l}\text { Bidirectional repeatability } \\
\text { of positioning at a position } R_{i}\end{array}$ & 12.5 & 9.7 & 6.7 & 11.7 & 16.0 & 7.3 & 14.1 & 9.3 & 11.8 \\
\hline
\end{tabular}

Retrospective analysis and communication with the positioner owner proves frequent overloading during the welding processes in these positions [17].

Results were satisfying; however, the higher values of unidirectional repeatability of positioning at position $R_{i} \downarrow$, comparing to $R_{i} \uparrow$ (clockwise) positions, are in Table 4 . We measure this effect within all counterclockwise positions. It might be due to the full impact of the gravitational force induced by the loading weight $(31.5 \mathrm{~kg})$ located in the vertical position and some backlash in the positioner mechanism (persistent unilateral load); the center of gravity aims directly down. The bi-directional repeatability of positioning at position $R_{i}$ as a correlation parameter confirms that the vertical forces have a more significant influence on the accuracy and repeatability of the positioner rotary axis but still do not exceed the standard guaranteed by the producer. The vertical component of force induced by the weight continues to move to target positions with an increment of $45^{\circ}$ and weakens while reaching horizontal positions. The mean bi-directional positional deviations at position $\bar{x}_{i}$ are in the graph (Fig.4.). The calculated value from the nine measures is in the range of -5.1 to $1.6 \mu \mathrm{m}$. 
Derived parameters of accuracy and repeatability of the positioner axis are in Table 5.

Table 5. Additional parameters of accuracy and repeatability of the positioner axis.

\begin{tabular}{lc}
\hline Parameter & $\mu \mathrm{m}$ \\
\hline Unidirectional accuracy of positioning of the axis A $\uparrow$ & 15.8 \\
Unidirectional accuracy of positioning of the axis A $\downarrow$ & 19.6 \\
Bi-directional accuracy of positioning of the axis A & 19.6 \\
Reversal value of the axis B & 5 \\
Mean reversal value of an axis $\bar{B}$ & 0.7 \\
Unidirectional repeatability of positioning R $\uparrow$ & 8.8 \\
Unidirectional repeatability of positioning R $\downarrow$ & 16 \\
Bi-directional repeatability of positioning R & 16 \\
Unidirectional systematic positional deviation of the axis & 7.6 \\
E $\uparrow$ & \\
Unidirectional systematic positional deviation of the axis & 7.1 \\
E $\downarrow$ & \\
Bi-directional systematic positional deviation of the axis E & 8.2 \\
Mean bi-directional positional deviation of the axis M & 6.6 \\
\hline
\end{tabular}

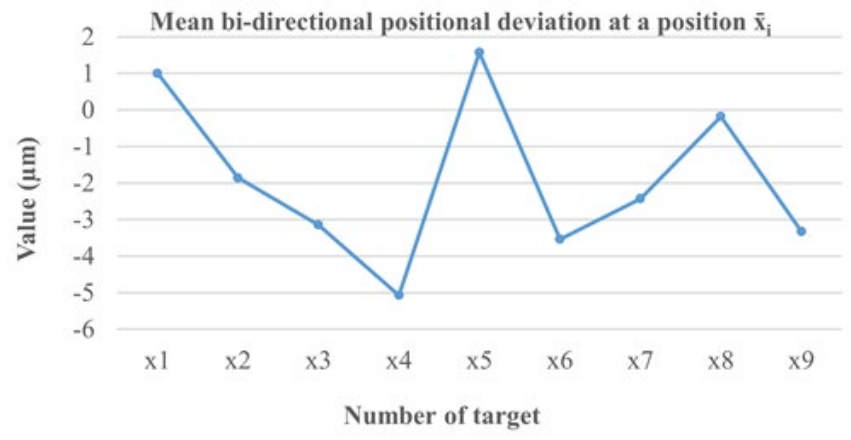

Fig.4. Mean bi-directional positional deviation at position $\bar{x}_{i}$.

The reversal value at position $B_{i}$ is in the graph (Fig.5.). The calculated value from the nine measures is in the range -3.9 to $5 \mu \mathrm{m}$.

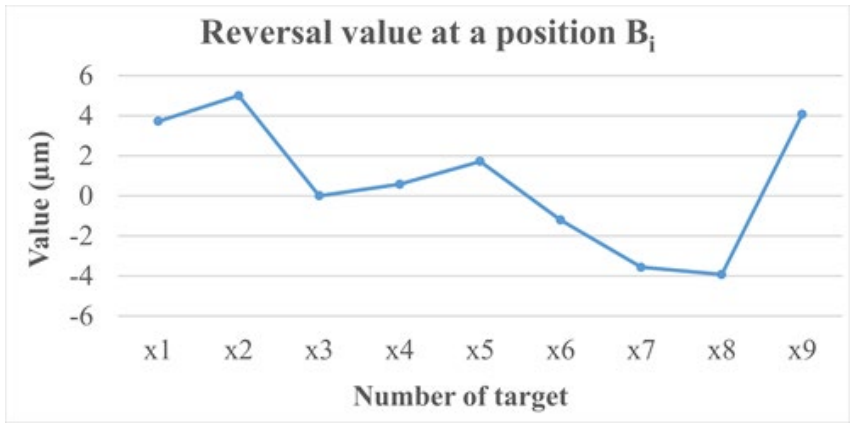

Fig.5. Reversal value at position $B_{i}$.

Estimating the unidirectional axis repeatability of positioning at a- $S_{i} \uparrow$ (uncertainty) position is in the graph (Fig.6.). The calculated value from the nine measures is in the range of 0.1 to $2.3 \mu \mathrm{m}$.

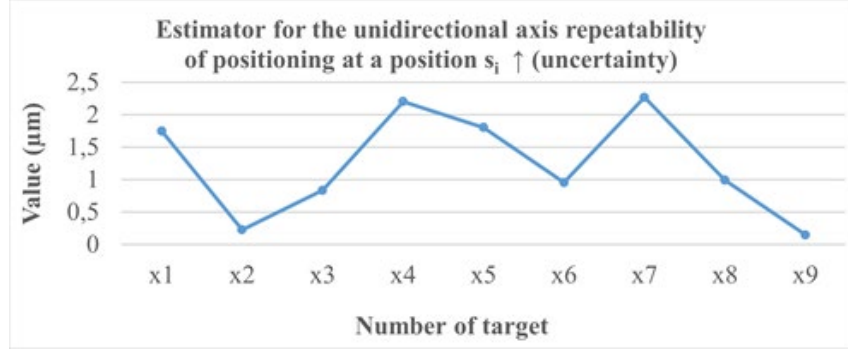

Fig.6. Estimator for the unidirectional axis repeatability of positioning at position $S_{i} \uparrow$ (uncertainty).

The estimator for the unidirectional axis repeatability of positioning at a- $S_{i} \downarrow$ (uncertainty) position is in the graph (Fig.7.). The calculated value from the nine measures is in the range of 1.7 to $4 \mu \mathrm{m}$.

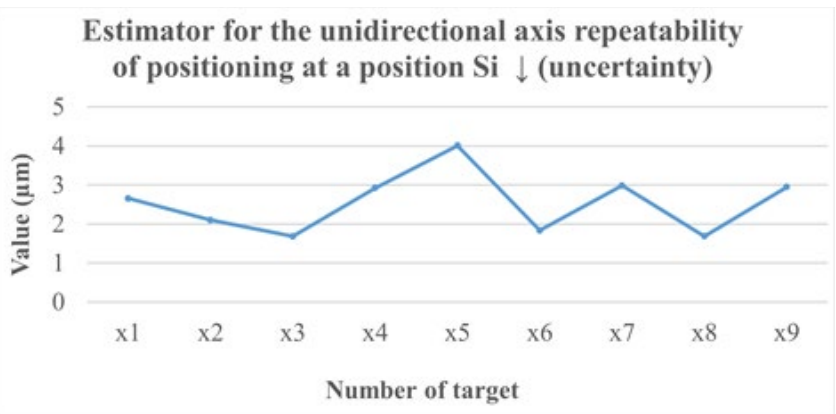

Fig.7. Estimator for the unidirectional axis repeatability of positioning at position $S_{i} \downarrow$ (uncertainty).

The Unidirectional repeatability of positioning at position $R_{i} \uparrow$ is in the graph (Fig.8.). The calculated value from the nine measures is in the range of 0.9 to $9.1 \mu \mathrm{m}$. 


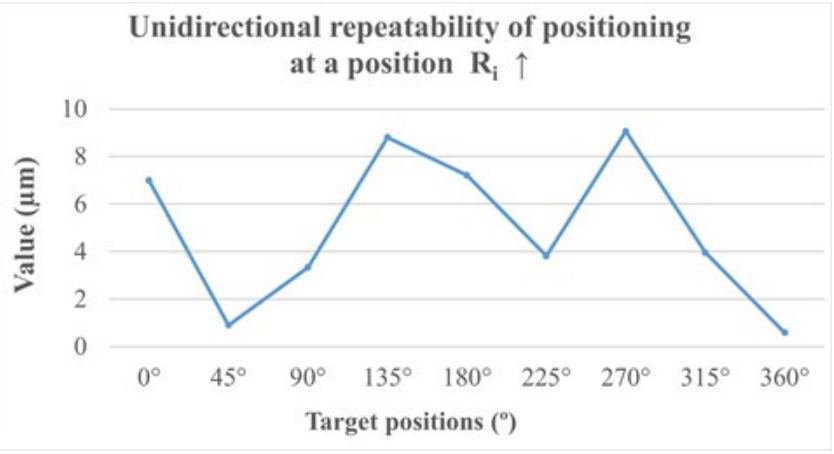

Fig.8. Unidirectional repeatability of positioning at position $R_{i} \uparrow$.

The Unidirectional repeatability of positioning at position $R_{i} \downarrow$ is in the graph (Fig.9.). The calculated value from the nine measures is in the range of 6.7 to $11.9 \mu \mathrm{m}$.

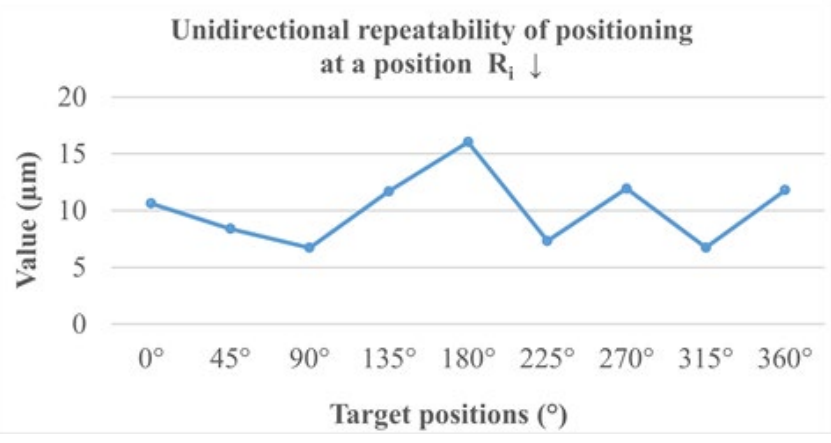

Fig.9. Unidirectional repeatability of positioning at position $R_{i} \downarrow$.

The bi-directional repeatability of positioning at position $R_{i}$ is in the graph (Fig.10.). The calculated value from the nine measures is in the range of 6.7 to $16 \mu \mathrm{m}$.



Fig.10. Bi-directional repeatability of positioning at position $R_{i}$.

None of the measured values exceed the maximal allowed value by the manufacturer $(0.05 \mathrm{~mm})$. The measurement followed by the calculations of necessary data confirmed that the bi-directional positional accuracy of positioning $A$ is $0.0196 \mathrm{~mm}$. When we compare it with the repeatable accuracy value given by the positioner manufacturer $(0.0196 \mathrm{~mm}<0.05 \mathrm{~mm})$, the positioner does not exceed the prescribed value by the manufacturer and is in the range. The repeatable accuracy of the robotic arm has a value of $+/-0.08 \mathrm{~mm}$ that assumes the prediction for sufficient accuracy of the whole measurement chain (robotic arm and positioner), and the repeatability for the welding does not exceed $+/-0.1 \mathrm{~mm}$.

\section{CONCLUSION}

The results of these measurements presented in the article allow us to draw some general conclusions based on the trends obtained. In comparison with requirements from catalog SPINEA, we can prove that accuracy demands successfully promote our experimental testing and parameter verification. It demonstrates usages of the methodology with particular emphasis on the ISO standard fundamentals and available software solutions. The positioner's initial accuracy and repeatability measurements show that the rotary axis meets the company prescribed accuracy. We ensured high accuracy of the HEIDENHAIN length gauge sensor over the entire measuring length.

\section{ACKNOWLEDGMENT}

This contribution is supported by the Slovak Research and Development Agency (grants number VEGA 1/0330/19) Research and design of algorithms and systems for the fusion of disparate data in multisensor architectures, project: University Science Park TECHNICOM for Innovation Applications Supported by Knowledge Technology, ITMS: 26220220182, supported by the Research \& Development Operational Program funded by the ERDF." And H2020 project: Manufacturing Industry Digital Innovation Hubs (MIDIH), reference no. 767498 .

\section{REFERENCES}

[1] Kopac, J., Pusavec, F., Kramar, D., Uhan, P. (2013). How to improve the positional accuracy of HSC machine tools. In 7th Brazilian Congress on Manufacturing Engineering. Associacao Brasileira de Engenharia e Ciencias Mecanicas, 10 p.

[2] Palencar, R., Halaj, M., Kurekova, E. (2007). Evaluation of the positional deviation of numerically controlled axes. Measurement Science Review, 7 (3), 27-30.

[3] Mostyn, V., Huczala, D., Moczulski, W., Timofiejczuk, A. (2020). Dimensional optimization of the robotic arm to reduce energy consumption. MM Science Journal, 2020, 3745-3753.

[4] Kurekova, E., Halaj, M., Palencar, R., Loebl, T. (2011). The positional deviation in two numerically controlled axes. In MEASUREMENT 2011: 8th International Conference on Measurement. Bratislava, Slovakia: IMS SAS, 88-91.

[5] Stejskal, T., Dovica, M., Duris, S., Durisova, Z., Palencar, J. (2019). Direct measurement of stationary objects' dimensions with Michelson type incremental laser interferometer. Journal of Physics: Conference Series, 1379, 012065.

[6] Schwenke, H., Knapp, W., Haitjema, H., Weckenmann, A., Schmitt, R., Delbressine, F. (2008). Geometric error measurement and compensation of machines-an update. CIRP Annals, 57 (2), 660-675. 
[7] Kwang, L., Shin, D., Yang, S. (2017). Parallelism error measurement for the spindle axis of machine tools by two circular tests with different tool lengths. The International Journal of Advanced Manufacturing Technology, 88, 2883-2887.

[8] International Organization for Standardization (ISO). (2006). Test code for machine tools - Part 2: Determination of accuracy and repeatability of positioning numerically controlled axes. ISO 2302:2006.

[9] Semjon, J., Hajduk, M., Janos, R., Vagas, M. (2013). Modular welding fixtures for robotic cells. Applied Mechanics and Materials, 309, 80-87.

[10] Stejskal, T., Kelemenova, T., Dovica, M., Demec, P., Stofa, M. (2016). Information contents of a signal at repeated positioning measurements of the coordinate measuring machine (CMM) by a laser interferometer. Measurement Science Review, 16 (5), 273-279.

[11] Fieger, T.V., Sattler, M.F., Witt, G. (2018). Developing laser beam welding parameters for the assembly of steel SLM parts for the automotive industry. Rapid Prototyping Journal, 24 (8), 1288-1295.

[12] Lira, I., Cargill, G. (2004). Uncertainty analysis of positional deviations of CNC machine tools. Precision Engineering, 28 (2), 232-239.
[13] Semjon, J., Hajduk, M., Sukop, M., Balaz, V., Pilat, Z., Sulik, M., Putala, J. (2016). Testing of parameters of proposed robotic wrist based on the precision modules. International Journal of Advanced Robotic Systems, 13 (5), 1-7.

[14] Semjon, J., Janos, R., Sukop, M., Tuleja, P., Hajduk, M., Jurus, O., Marcinko, P., Virgala, I., Vagas, M. (2020). Verification of the UR5 robot's properties after a crash caused by a fall of a transferred load from a crane. International Journal of Advanced Robotic Systems, 17 (1), 1-9.

[15] Semjon, J., Janos, R., Sukop, M., Vagas, M., Varga, J., Hroncova, D., Gmiterko, A. (2017). Mutual comparison of developed actuators for robotic arms of service robots. International Journal of Advanced Robotic Systems, 14 (6), 1-8.

[16] Huo, D., Cheng, K., Wardle, F. (2009) Design of precision machines. In Machining Dynamics: Fundamentals, Applications, and Practices. Springer, 283-321. ISBN 978-1-84628-367-3.

[17] SPINEA Technologies. RotoSpin - High precision rotary modules. https://www.spinea.com/en/products/ rotospin/rotospin-a. 